\title{
Distraction and the auditory attentional blink
}

\author{
János Horváth • Annamária Burgyán
}

Published online: 24 December 2010

(C) Psychonomic Society, Inc. 2010

\begin{abstract}
When two target stimuli (T1 and T2) are presented in close succession within a stimulus sequence, detection or discrimination performance for $\mathrm{T} 2$ is often impaired; this phenomenon is called the attentional blink (AB). Growing evidence suggests that the $\mathrm{AB}$ is present not only for visual, but also for auditory stimuli. Because sounds have a strong potential to attract attention when they are presented after longer silent intervals or in a sequence from which they stand out because of their rare or novel physical features, it seems possible that the involuntary and excessive allocation of attention to $\mathrm{T} 1$ (distraction) may contribute to the $\mathrm{AB}$. By varying the saliency of $\mathrm{T} 1$, we showed that distraction does contribute to the auditory $\mathrm{AB}$ effect, but the contribution is short-lived: Only stimuli immediately following $\mathrm{T} 1$ are affected.
\end{abstract}

Keywords Attention - Attentional blink · Orienting ·

Distraction · Audition · Stimulus-driven $\cdot$ Salience

\section{Introduction}

When two target stimuli (the first target [T1] and the second target [T2]) are presented in close succession within a stimulus sequence providing masking, detection or discrimination performance for T2 is often impaired; this phenomenon is called the attentional blink (AB). Whereas

J. Horváth $(\bowtie)$

Institute for Psychology, Hungarian Academy of Sciences,

Szondi u. 83/85, P.O.B. 398, Budapest 1394, Hungary

e-mail: horvath@cogpsyphy.hu

A. Burgyán

Faculty of Education and Psychology, Eötvös Loránd University,

Budapest, Hungary numerous studies have investigated the $\mathrm{AB}$ in the visual domain (for a recent review, see Dux \& Marois, 2009), relatively few studies have addressed whether the $A B$ is also present in other modalities. In the auditory domain, results resembling those obtained in visual paradigms have been reported by a number of studies (e.g., Duncan, Martens, \& Ward, 1997; Mondor, 1998; Shen \& Mondor, 2008; Vachon \& Tremblay, 2008; Van der Burg, Olivers, Bronkhorst, Koelewijn, \& Theeuwes, 2007). Whereas the $\mathrm{AB}$ is probably the sum of different effects (Dux \& Marois, 2009), most explanations of the $A B$ have been based on the assumption that $\mathrm{AB}$ is caused by interference from the voluntary, task-relevant processing of $\mathrm{T} 1$. In the present study, we investigated, in an auditory paradigm, whether (auditory) AB was caused (at least in part) by the exogenous, saliency-dependent allocation of attentional resources to $\mathrm{T} 1$.

It is well known that auditory stimuli attract attention depending on their context. Notably, sounds presented after longer silent periods or those that stand out from a series of sounds because of their rare or novel physical features or because they violate a temporal regularity of the sequence have a strong potential to draw attention to themselves (see, e.g., Cherry, 1953; Näätänen, 1990; Schröger, 1997). The processes leading to exogenous auditory attention capture have been extensively studied with the event-related potential (ERP) technique. Briefly summarized, this line of research provides evidence that incoming sounds are processed by a sensory filter, which "absorbs" predictable stimuli; that is, the sensory filter processes sounds fitting the given auditory context without involving attentional, capacity-limited resources. Infrequent, temporally unpredictable sensory events, on the other hand, lead to the allocation of attentional resources to the processing of the event (for a summary, see, e.g., Escera \& Corral, 2007; Rinne, Särkkä, Degerman, Schröger, \& Alho, 2006). This 
stimulus-driven attention capture mechanism is also active in situations in which the stimuli are attended: Taskirrelevant sounds or task-relevant sounds with rare taskirrelevant feature variations also initiate a change in the attentional state that is detrimental to the task-relevant processing; that is, we get distracted (see, e.g., Horváth, Winkler, \& Bendixen, 2008; Roeber, Widmann, \& Schröger, 2003; Schröger \& Wolff, 1998). It has been shown that the distracted state persists for at least $150 \mathrm{~ms}$ after the triggering event: When a task-relevant auditory event is closely (by $150 \mathrm{~ms}$ ) preceded by a rare, salient, but task-irrelevant sound event, the N1 ERP (for a review, see Näätänen \& Picton, 1987) elicited by the task-relevant event is reduced, suggesting that after the salient event, the allocation of input attention becomes temporarily suboptimal for the processing of the task-relevant event (Horváth \& Winkler, 2010). Although the attentional changes can be suppressed if rare sounds are cued just before their delivery, only processes upstream from the sensory filter are affected by direct preparatory activities (Sussman, Winkler, \& Schröger, 2003). It has to be noted that topdown influences can affect the sensory filter in indirect ways: Sussman, Winkler, Huotilainen, Ritter, and Näätänen (2002) showed that if the auditory input has a temporal structure that is difficult to find at first hearing, supplying information on the "hidden" structure leads to the reorganization of the auditory input into a predictable pattern, in which previously outstanding tones become a natural part of a longer repeating pattern and, therefore, no longer stand out. In summary, the auditory system automatically provides a signal that "highlights" auditory events that stand out from the established stimulus context. It seems plausible that tasks involving the detection of outstanding sounds rely on this signal and the mechanisms triggering the allocation of attention to such sounds.

The situations in which auditory distraction occurs cover most setups in which a robust auditory $\mathrm{AB}$ can be observed (see the references above). Setups in which stimuli do not allow for the establishment of a more-or-less regular or homogeneous auditory context, in which target status cannot be determined on the basis of primary physical feature differences (e.g., detecting various spoken digits in a mixture of various spoken letters), generally do not produce a robust auditory AB (Arnell \& Jenkins, 2004; Koelewijn, Van der Burg, Bronkhorst, \& Theeuwes, 2008; Potter, Chun, Banks, \& Muckenhoupt, 1998; but see Tremblay, Vachon, \& Jones, 2005, Experiment 3; Martens, Johnson, Bolle, \& Borst, 2009, Experiment 2). In the design most commonly used to elicit a robust $\mathrm{AB}$, the two targets are presented among many nontarget stimuli in the context of short stimulus sequences. Because the sequence of nontargets preceding the targets establishes a stimulus context from which targets stand out, the sensory filter described above automatically "highlights" these sounds, which leads to an excessive allocation of attention to these targets. Therefore, it seems possible that $\mathrm{AB}$ effects found in similar setups are, at least in part, due to this bottom-up mechanism triggered by T1. Because distraction effects generally increase with the saliency of the rare sound (see, e.g., Berti, Roeber, \& Schröger, 2004; Escera, Yago, \& Alho, 2001; Yago, Corral, \& Escera, 2001), we assessed the effect of distraction by manipulating the magnitude of $\mathrm{T} 1$ salience in relation to nontarget tones. We hypothesized that $\mathrm{AB}$ effects should be larger for higher $\mathrm{T} 1$ salience. Closely following the design used by Vachon and Tremblay (2005), we used detection tasks: T1 was a tone separated by its tonal frequency from nontargets, whereas T2 was a complex tone comprising components in the frequency range of the nontarget tones.

\section{Experiment 1}

\section{Method}

Eighteen paid volunteers (ten of them female; age 1925 years) reporting normal hearing status participated in Experiment 1. All the participants gave written informed consent after the experimental procedures were explained to them. The participants were seated in a comfortable chair in a sound-attenuated room during the experiment. Sounds were presented through a headphone (HD-600, Sennheiser, Germany). The intensity level of the stimuli was individually adjusted to a 40-dB sensation level (above hearing threshold level).

Three types of tones were presented. Nontarget sounds were sinusoid tones with random frequencies in the 440 $1760 \mathrm{~Hz}$ range in 1-semitone (ST) steps. T1s were sinusoid tones with frequencies of 3951 and $2960 \mathrm{~Hz}$ (14 and 9 ST higher than the highest nontarget tone); these are referred to as $14 S T-T 1$ and $9 S T-T 1$, respectively, in the following. $T 2$ was a complex tone with three sinusoid components, separated by +7 and +12 ST, as compared with the lowest component (with relative intensities of -12 and $-24 \mathrm{~dB}$, respectively). The frequency of the lowest $\mathrm{T} 2$ component was random in the range of $440-880 \mathrm{~Hz}$, with 1-ST steps. The signal energy of T2 was the same as that of the nontarget tones. The duration of the tones was $30 \mathrm{~ms}$ (including 2.5-/2.5-ms linear rise/fall times).

On each trial, a sequence of tones was presented. The sequences (see Fig. 1) started with 7-15 nontarget tones (randomly, with uniform distribution). T1 could be delivered in the position following this initial sequence. T2 could be delivered in the next (lag 1), the third (lag 3), or the 7th (lag 7) position after T1. The positions between T1 and $\mathrm{T} 2$ positions were filled with nontarget tones. When no 
Fig. 1 The schematic temporal structure of the tone sequences presented on each trial in both experiments. Target stimuli (T1 and T2) are presented in gray. T1s were pure tone stimuli separated by 9 or 14 semitones (9ST-T1 and 14ST-T1) from the frequency range of the standard tones $(\mathrm{S})$, whereas $\mathrm{T} 2 \mathrm{~s}$ were complex tones with components fully within the frequency range of the standard tones

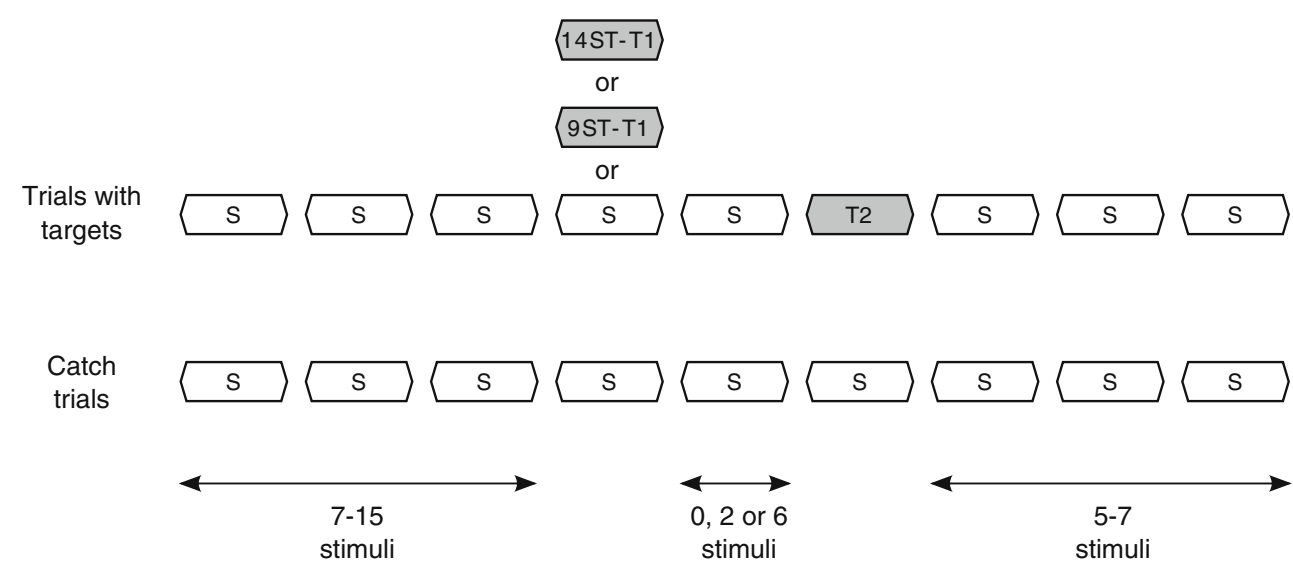

T1 or T2 was delivered in a given position, a nontarget tone was presented instead. The sequence was concluded by 5-7 nontarget tones (randomly, with uniform distribution). The onset-to-onset interstimulus interval was $90 \mathrm{~ms}$.

The participants' task was to report specifically whether they had heard a T1 ("high") or T2 ("complex") in the sequence; they were informed that it was possible to hear both T1 and T2, only T1, only T2, or neither of them. The discrimination of the two types of T1s was not part of the task. Participants were also asked to report what they had heard and to refrain from guessing. The experimenter started the next trial after the report. No feedback about the accuracy of the reports was provided.

There were ten different types of trials/sequences. Unknown to the participants, nine of these contained a T2 and resulted from the permutations of the $\mathrm{T} 1$ stimulus (14ST-T1 present, 9ST-T1 present, or no T1 present) and lag $(1,3$, or 7$)$ factors. The tenth type of sequence-the catch trial-contained neither T1 nor T2. (That is, overall, six of the ten sequence types contained a T1.) The presentation of catch trials was necessary because, in this arrangement, some participants might discover that T2 was present on most trials (90\%), which might lead to reports that reflect this knowledge, and not the participants' percept regarding T2. If a participant reported the presence of T2 on a high proportion of catch trials, it would indicate that responses were based not on the actual percept, but on guessing or response bias. Each experimental block contained two of each type of trial, which were presented in random order, for a total of 20 trials per block. Overall, 20 blocks were presented, separated by short breaks as needed, for 40 instances of each trial/sequence type during the experiment.

Before the experiment, participants were familiarized with the stimulus material and target events. First, 20 sequences containing a single 9 ST-T1 with $50 \%$ probability were presented. If the participant had difficulty in discriminating sequences with $\mathrm{T} 1$ presence and absence, sequences containing a single 14 ST-T1 with $50 \%$ probability were presented. This was sufficient in all cases to demonstrate the difference between the T1 and the standards, and it was followed by training with sequences containing a single 9ST-T1 with $50 \%$ probability (one to three blocks of 20 sequences). To demonstrate the difference between pure and complex tones, a six-element alternating sequence of single pure and complex tones was presented, which was followed by a random sequence of pure and complex tones (50\%-50\%), for which participants had to report whether they heard a pure or a complex tone. Then one to three blocks of 20 sequences containing a single T2 with $50 \%$ probability were presented.

Hit rates for $\mathrm{T} 2$ on those trials on which the presence or absence of a T1 was correctly detected were submitted to a stimulus $(14 \mathrm{ST}-\mathrm{T} 1,9 \mathrm{ST}-\mathrm{T} 1$, no T1) $\times \operatorname{lag}(1,3,7)$ repeated measures analysis of variance (ANOVA). Similarly, hit rates for $\mathrm{T} 1$ on those trials on which the presence of T2 was correctly detected were submitted to a stimulus (14ST-T1 and 9ST-T1) $\times$ lag $(1,3,7)$ repeated measures ANOVA. Greenhouse-Geisser correction was applied as appropriate. Partial eta-squared values are reported. All significant effects are reported.

\section{Results and discussion}

The mean correct response rate (or correct rejection rate) combined for T1 and T2 for catch trials (on which neither T1 nor T2 was present) was 97\% $\pm 1 \%$ (range: $85 \%-100 \%$ ). False alarms on catch trials resulted from the report of either T1 $(2 \% \pm 3 \%$; range: $0.0 \%-7.5 \%)$ or T2 (1\% $\pm 2 \%$; range: $0 \%-7.5 \%$, but not both. This suggests that participants generally refrained from guessing the presence of targets; therefore, no participant's data were rejected from the analyses.

The ANOVA of the hit rates for $\mathrm{T} 2$ on the trials on which the presence or absence of $\mathrm{T} 1$ was correctly reported (see Fig. 2), showed a stimulus main effect, $F(2,34)=$ 74.57, $\varepsilon=.67, \eta_{\mathrm{p}}=.81, p<.001$, a lag main effect, $F(2,34)=51.87, \varepsilon=.95, \eta_{\mathrm{p}}=.75, p<.001$, and $\mathrm{a}$ 


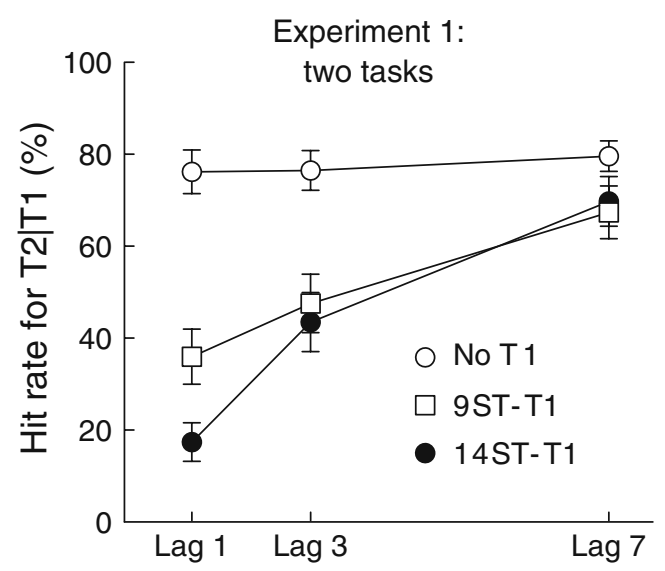

Fig. 2 Mean T2 detection hit rates, with standard errors, for those trials on which the presence or absence of $\mathrm{T} 1$ was correctly reported in Experiment 1

stimulus $\times$ lag interaction, $F(4,68)=26.03, \varepsilon=.59, \eta_{\mathrm{p}}=.62$, $p<.001$. A Tukey HSD test showed that hit rates increased significantly with increasing lag for the 14ST-T1 and 9ST-T1 trials $(p s<.001)$, but no significant difference was found for the no-T1 trials. At lag 1, all three hit rates differed significantly $(p \mathrm{~s}<.001)$. At lag 3 , hit rate was higher on no-T1 trials, as compared with the other two trial types $(p s<.001)$. At lag 7, hit rate was higher on no-T1 than on 9ST-T1 trials $(p<.05)$.

The ANOVA of the hit rates for the detection of $\mathrm{T} 1$ on trials on which $\mathrm{T} 2$ was present and its presence was correctly reported (see Fig. 3) showed a stimulus main effect, $F(1,17)=20.55, \eta_{\mathrm{p}}=.55, p<.001$, showing that the detection of 9ST-T1 was more difficult than that of the 14ST-T1. There was also a lag main effect, $F(2,34)=$ 12.66, $\varepsilon=.56, \eta_{\mathrm{p}}=.43, p<.001$. A Tukey HSD test showed that hit rate was significantly lower for lag 1 , than for lag 3 or $7(p s<.001)$.

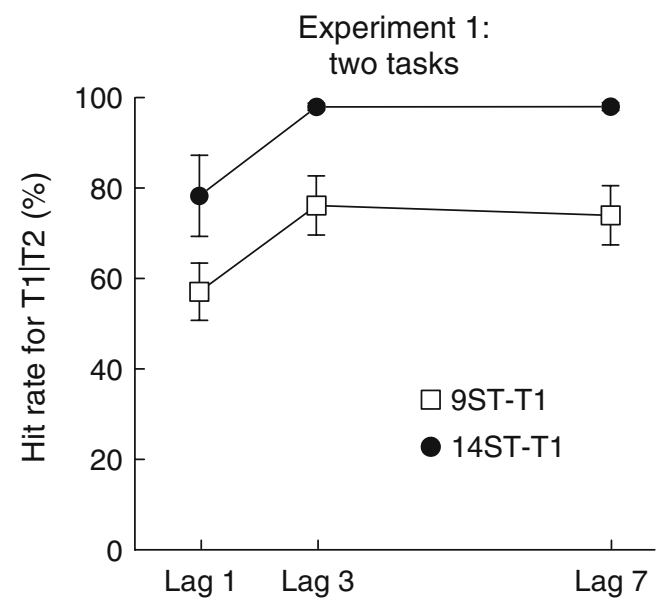

Fig. 3 Mean T1 detection hit rates, with standard errors, for those trials on which $\mathrm{T} 2$ was present and successfully detected in Experiment 1
Experiment 1 successfully replicated the general $\mathrm{AB}$ findings from previous studies: Following T1, the T2 detection deficit was present at short lags, but it diminished with longer lags (Fig. 2). Importantly, the results indicate that there are at least two effects involved in the generation of $A B$ in the present paradigm. The first is dependent on the saliency of the T1 stimulus in the context of the nontarget sequence and is relatively short-lived: The modulatory effect of saliency was present only for the immediately following T2, but not for T2s at lag 3 or lag 7. This saliency-dependent effect cannot be explained by forward masking: Because the effect of forward masking should be larger when the mask and the following tone are closer in their frequencies, forward masking should produce just the opposite of what was observed (i.e., the 9ST-T1 should mask T2 more effectively than should the 14ST-T1). Note that the results show an interference between the two types of targets in those trains where $\mathrm{T} 2$ was presented immediately after $\mathrm{T} 1$, as compared with the other two lags (see Fig. 3). This interference effect, however, did not significantly differ between the levels of $\mathrm{T} 1$ saliency.

Whereas T1 saliency modulated T2 detection performance at lag 1, this modulation was no longer observed at $\operatorname{lag} 3$, which suggests that there was a second, non-saliencydependent effect, which was present after both T1s for a longer period of time, that is commensurate to the duration of the auditory $\mathrm{AB}$ observed in previous studies.

Although the results of Experiment 1 are in line with the assumption that stimulus-driven bottom-up attention capture mechanisms (distraction) contribute to auditory $\mathrm{AB}$, it could be still argued that the short-lived saliency-dependent effect is brought about by the task relevancy of the T1 stimuli, and not by their saliency per se. To investigate this possibility, we conducted Experiment 2, in which participants performed only the complex tone ("T2") detection task and were not informed about the presence of the higher-frequency pure tones ("T1").

\section{Experiment 2}

Method

Eighteen paid volunteers (11 of them female; age 18-26 years) reporting normal hearing status participated in Experiment 2. None of them had participated in Experiment 1. All the participants gave written informed consent after the experimental procedures were explained to them. The experimental procedures were identical to those in Experiment 1 , except for the instructions, in which the high pure tones (T1s in Experiment 1) were not mentioned at all; also, only the familiarization procedure with the complex tones was carried out. Participants were instructed to report after 
each sequence whether they had heard a complex tone in the sequence or not. Although only the complex tone was task relevant in Experiment 2, for the sake of simplicity, we refer to the salient high-frequency tones as T1s and to the complex target tone as $\mathrm{T} 2$ in the following.

\section{Results and discussion}

One participant's data were excluded from the analyses because the correct rejection rate on the catch trials (on which neither $\mathrm{T} 1$ nor $\mathrm{T} 2$ was present) was only $60 \%$, which suggests guessing or response bias. The remaining 17 participants' mean correct response rate on the catch trials was $96 \% \pm 1 \%$ (range: $85 \%-100 \%$ ).

The ANOVA of the hit rates for T2 (see Fig. 4), showed a stimulus main effect, $F(2,32)=42.77, \varepsilon=.72, \eta_{\mathrm{p}}=.73$, $p<.001$, a lag main effect, $F(2,32)=50.52, \varepsilon=.76, \eta_{\mathrm{p}}=$ $.75, p<.001$, and a stimulus $\times$ lag interaction, $F(4,64)=$ $18.24, \varepsilon=.82, \eta_{\mathrm{p}}=.53, p<.001$. A Tukey HSD test showed that hit rates increased significantly with increasing lag for the 14ST-T1 trials $(p \mathrm{~s}<.001)$. For the 9ST-T1 trials, the hit rate was higher at lag 3 and lag 7 than at lag 1 ( $p \mathrm{~s}<.001)$, but no significant difference was found between lag 3 and lag 7 trials. There were no significant differences between no-T1 trials. At lag 1 and lag 3 , all three hit rates differed significantly ( $p \mathrm{~s}<.001$ at lag 1 , and $p \mathrm{~s}<.05$ at lag 3). At lag 7, no significant differences were found.

These results show that the detectability of the complex target tones decreased when they were closely preceded by salient tones, even if the salient tones were task irrelevant; this effect was present at lags 1 and 3. Moreover, this effect was modulated by $\mathrm{T} 1$ saliency: T2s following more salient T1s were less easily detectable. Although this pattern is very similar to that obtained in Experiment 1, there is also a difference: In Experiment 1, T1 saliency modulated T2 detectability only at lag 1 , but not at lag 3 . A plausible

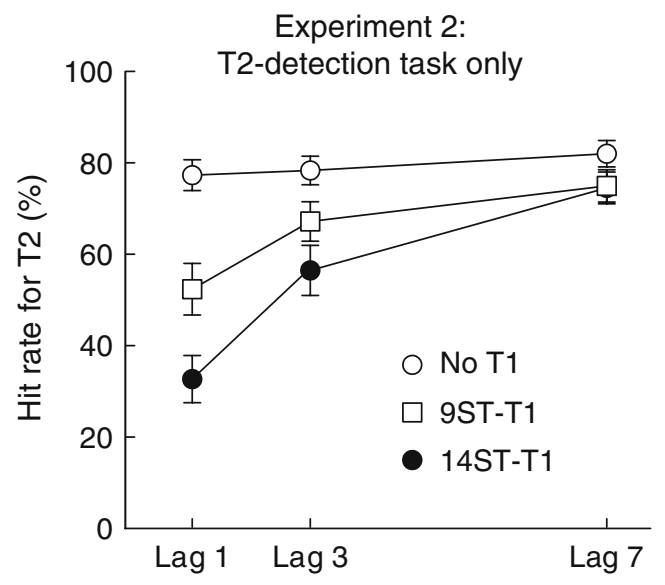

Fig. 4 Mean T2 detection hit rates, with standard errors, in Experiment 2 explanation for this difference is that in the dual detection task situation, explicit knowledge about salient $\mathrm{T} 1 \mathrm{~s}$ and their task relevancy may have enabled participants to temporally constrain the T1-saliency-related attentional effect, whereas in Experiment 2, this was not possible.

\section{General discussion}

The present study investigated the role of stimulus-driven attention capture (distraction) in the prototypical paradigm used to elicit a robust auditory AB. The results show that salient tones embedded in a tone sequence draw attentional resources away from the processing of closely following stimuli, even if the salient tones are task irrelevant.

Although input attention was drawn away by a salient tone, attention was essentially restored by about $270 \mathrm{~ms}$ from the onset of the distracting tone (lag 3) when salient tones were task relevant (Experiment 1). On the other hand, when salient tones were task irrelevant, $\mathrm{T} 2$ detection performance was still modulated by T1 saliency at lag 3 (Experiment 2). The lack of a similar modulation at lag 3 in Experiment 1 suggests that explicit instructions regarding T1 may enable participants to form a more structured percept of the target sounds within the sequence. Nonetheless, this hypothetical structuring effort has its limits: T1 saliency still modulated $\mathrm{T} 2$ performance at lag 1 in Experiment 1.

When the dual detection task was performed (Experiment 1), T2 detection rate was decreased by preceding T1s at lag 3, but no saliency modulation was observed. This suggests that a further, non-saliency-dependent effect was also present in this experiment. Because of its longerlasting effect, commensurate with the duration of $\mathrm{AB}$ effects observed in previous studies, this effect is probably the $A B$ effect brought about by having to perform two acts of target detection in a row in a short period of time. That is, the present study demonstrates that distraction triggered by salient stimuli may well overlap a "genuine" AB effect.

Whereas the distraction effect triggered by $\mathrm{T} 1$ is apparently not sufficient to fully explain $\mathrm{AB}$ in the auditory modality, it may explain why lag 1 sparing - that is, a lack of performance deficit in the position immediately following T1 (Potter et al., 1998) - might not be as easily observable in auditory $\mathrm{AB}$ studies as in visual paradigms (see, e.g., Arnell \& Jenkins, 2004). In the visual modality, lag 1 sparing is generally explained by assuming that the detection of $\mathrm{T} 1$ leads to transient attentional enhancement, which improves performance for a closely following T2 (Nieuwenstein, Van der Burg, Theeuwes, Wyble, \& Potter, 2009). This effect, however, seems to be constrained to visual paradigms in which exogenous attention capture by T1 does not occur. In setups with salient, task-irrelevant 
T1s, a short-lived $\mathrm{AB}$ with no lag 1 sparing can also be observed (Spalek, Falcon, \& Di Lollo, 2006, Experiment 4) that is similar to that measured in the present auditory paradigm. It seems that in the auditory modality, the transient attentional change triggered by a salient target impacts performance for closely following stimuli. Indeed, because $\mathrm{T} 1$ saliency affected the $\mathrm{T} 2$ detection deficit at lag 1 in the present experiments, it is possible that the deficit at this position was caused exclusively by the T1-saliencydependent effect. This interpretation is in line with the results of a between-experiments comparison of $\mathrm{T} 2$ detection performance for lag 1 (one-way ANOVA with experiment as a between-subjects and lag as a withinsubjects factor), which showed no significant effects including the experiment factor.

We started out with an observation about the auditory $\mathrm{AB}$ literature, suggesting that whereas a robust auditory $\mathrm{AB}$ can be found mostly in experiments utilizing a salient $\mathrm{T} 1$, studies using paradigms with nonsalient $\mathrm{T} 1 \mathrm{~s}$ generally do not produce a robust auditory $\mathrm{AB}$. There are, however, some counterexamples: Tremblay et al. (2005, Experiment 3) and Martens et al. (2009, Experiment 2) presented target syllables within a mixture of different syllables and found marked $A B$ effects. A speculative explanation accommodating these two findings is based on the fact that, in both experiments, T1 was presented at a fixed position within the stimulus sequences. Because of this, participants may have "focused" their attention on this temporal position (Nobre, Correa, \& Coull, 2007). This hypothetical voluntary mobilization of attentional resources is similar to the saliency-related attentional change, in the sense that both "highlight" $\mathrm{T} 1$ or the $\mathrm{T} 1$ position relative to nontargets. Note that this selective mobilization is not related to the evaluation of $\mathrm{T} 1$ in task-related terms per se (i.e., whether it is a target or a nontarget); rather, it is related to the circumstances of its presentation (its saliency in one case and an attentional strategy, based on its temporal predictability, in the other case). Therefore, we suggest that the (voluntary or involuntary) mobilization of attentional resources enhancing $\mathrm{T} 1$, relative to the nontargets, may be a necessary condition for the elicitation of the auditory AB. Whereas this may be a necessary condition, it does not seem to be sufficient: A number of studies have shown that auditory $\mathrm{AB}$ does not occur (even when $\mathrm{T} 1$ is salient) when $\mathrm{T} 2$ is followed very closely $(10 \mathrm{~ms})$ or only after a relatively long delay ( $350 \mathrm{~ms}$ or more) by nontarget tones (Shen \& Mondor, 2006; Vachon \& Tremblay, 2005, 2006).

In summary, the present experiments showed that in the prototypical auditory paradigm used to elicit a robust $A B$, salient T1 stimuli bring about two overlapping effects, both of which impair T2 task performance: They trigger a T1-salience-dependent, short-lived distraction effect and a longer lasting "genuine" AB (caused by having to perform two tasks in close succession).

Acknowledgements We thank István Czigler and two anonymous reviewers for valuable comments on the study. The research has received funding from the European Community's Seventh Framework Programme (under grant agreement PERG04-GA-2008-239393).

\section{References}

Arnell, K. M., \& Jenkins, R. (2004). Revisiting within-modality and cross-modality attentional blinks: Effects of target-distractor similarity. Perception \& Psychophysics, 66, 1147-1161.

Berti, S., Roeber, U., \& Schröger, E. (2004). Bottom-up influences on working memory: Behavioral and electrophysiological distraction varies with distractor strength. Experimental psychology, 51, 249-257. doi:10.1027/1618-3169.51.4.249

Cherry, E. C. (1953). Some experiments on the recognition of speech, with one and with two ears. Journal of the Acoustical Society of America, 25, 975-979. doi:10.1121/1.1907229

Duncan, J., Martens, S., \& Ward, R. (1997). Restricted attentional capacity within but not between sensory modalities. Nature, 387, 808-810. doi:10.1038/42947

Dux, P. E., \& Marois, R. (2009). The attentional blink: A review of data and theory. Attention, Perception, \& Psychophysics, 71, 1683-1700. doi:10.3758/APP.71.8.1683

Escera, C., \& Corral, M. J. (2007). Role of mismatch negativity and novelty-P3 in involuntary auditory attention. Journal of Psychophysiology, 21, 251-264. doi:10.1027/0269-8803.21.34.251

Escera, C., Yago, E., \& Alho, K. (2001). Electrical responses reveal the temporal dynamics of brain events during involuntary attention switching. European Journal of Neuroscience, 14, 877-883. doi:10.1046/j.0953-816x.2001.01707.x

Horváth, J., \& Winkler, I. (2010). Distraction in a continuousstimulation detection task. Biological Psychology, 83, 229-238. doi:10.1016/j.biopsycho.2010.01.004

Horváth, J., Winkler, I., \& Bendixen, A. (2008). Do N1/MMN, P3a, and RON form a strongly coupled chain reflecting the three stages of auditory distraction? Biological Psychology, 79, 139147. doi:10.1016/j.biopsycho.2008.04.001

Koelewijn, T., Van der Burg, E., Bronkhorst, A., \& Theeuwes, J. (2008). Priming T2 in a visual and auditory attentional blink task. Perception \& Psychophysics, 70, 658-666. doi:10.3758/ PP.70.4.658

Martens, S., Johnson, A., Bolle, M., \& Borst, J. (2009). A quick visual mind can be a slow auditory mind: Individual differences in attentional selection across modalities. Experimental Psychology, 56, 33-40. doi:10.1027/1618-3169.56.1.33

Mondor, T. (1998). A transient processing deficit following selection of an auditory target. Psychonomic Bulletin \& Review, 5, 305311.

Näätänen, R. (1990). The role of attention in auditory information processing as revealed by event-related potentials and other brain measures of cognitive function. Behavioral and Brain Sciences, 13, 201-288.

Näätänen, R., \& Picton, T. W. (1987). The N1 wave of the human electric and magnetic response to sound: A review and an analysis of the component structure. Psychophysiology, 24, 375425. doi:10.1111/j.1469-8986.1987.tb00311.x

Nieuwenstein, M., Van der Burg, E., Theeuwes, J., Wyble, B., \& Potter, M. (2009). Temporal constraints on conscious vision: On the ubiquitous nature of the attentional blink. Journal of Vision, 9, 1-14. doi:10.1167/9.9.18 
Nobre, A., Correa, A., \& Coull, J. (2007). The hazards of time. Current Opinion in Neurobiology, 17, 465-470. doi:10.1016/j. conb.2007.07.006

Potter, M. C., Chun, M. M., Banks, B. S., \& Muckenhoupt, M. (1998). Two attentional deficits in serial target search: The visual attentional blink and an amodal task-switch deficit. Journal of Experimental Psychology: Learning, Memory, and Cognition, 24, 979-992. doi:10.1037/0278-7393.24.4.979

Rinne, T., Särkkä, A., Degerman, A., Schröger, E., \& Alho, K. (2006). Two separate mechanisms underlie auditory change detection and involuntary control of attention. Brain Research, 1077, 135-143. doi:10.1016/j.brainres.2006.01.043

Roeber, U., Widmann, A., \& Schröger, E. (2003). Auditory distraction by duration and location deviants: A behavioral and event-related potential study. Cognitive Brain Research, 17, 347-357. doi:10.1016/S0926-6410(03)00136-8

Schröger, E. (1997). On the detection of auditory deviations: A preattentive activation model. Psychophysiology, 34, 245-257. doi:10.1111/j.1469-8986.1997.tb02395.x

Schröger, E., \& Wolff, C. (1998). Behavioral and electrophysiological effects of task-irrelevant sound change: A new distraction paradigm. Cognitive Brain Research, 7, 71-87. doi:10.1016/ S0926-6410(98)00013-5

Shen, D., \& Mondor, T. A. (2006). Effect of distractor sounds on the auditory attentional blink. Perception \& Psychophysics, 68, 228 243.

Shen, D., \& Mondor, T. A. (2008). Object file continuity and the auditory attentional blink. Perception \& Psychophysics, 70, 896915. doi:10.3758/PP.70.5.896

Spalek, T. M., Falcon, L. J., \& Di Lollo, V. (2006). Attentional blink and attentional capture: Endogenous versus exogenous control over paying attention to two important events in close succession. Perception \& Psychophysics, 68, 674-684.

Sussman, E., Winkler, I., Huotilainen, M., Ritter, W., \& Näätänen, R. (2002). Top-down effects can modify the initially stimulus-driven auditory organization. Cognitive Brain Research, 13, 393-405. doi:10.1016/S0926-6410(01)00131-8

Sussman, E., Winkler, I., \& Schröger, E. (2003). Top-down control over involuntary attention switching in the auditory modality. Psychonomic Bulletin \& Review, 10, 630-637.

Tremblay, S., Vachon, F., \& Jones, D. M. (2005). Attentional and perceptual sources of the auditory attentional blink. Perception \& Psychophysics, 67, 195-208.

Vachon, F., \& Tremblay, S. (2005). Auditory attentional blink: Masking the second target is necessary, delayed masking is sufficient. Canadian Journal of Experimental Psychology, 59, 279-286. doi:10.1037/h0087480

Vachon, F., \& Tremblay, S. (2006). Delayed masking and the auditory attentional blink: A test for retrieval competition and bottleneck models. Experimental Psychology, 53, 182-190. doi:10.1027/ 1618-3169.53.3.182

Vachon, F., \& Tremblay, S. (2008). Modality-specific and amodal sources of interference in the attentional blink. Perception \& Psychophysics, 70, 1000-1015. doi:10.3758/PP.70.6.1000

Van der Burg, E., Olivers, C. N., Bronkhorst, A. W., Koelewijn, T., \& Theeuwes, J. (2007). The absence of an auditory-visual attentional blink is not due to echoic memory. Perception \& Psychophysics, 69, 1230-1241.

Yago, E., Corral, M. J., \& Escera, C. (2001). Activation of brain mechanisms of attention switching as a function of auditory frequency change. NeuroReport, 12, 4093-4097. doi:10.1097/ 00001756-200112210-00046 